\title{
The causes of low adherence in patients with antihypertensive therapy
}

\author{
K. Spalva ${ }^{1}$, I. Urtane ${ }^{2}$, K. Puke ${ }^{1}$, S. Berzina ${ }^{2}$, and D. Bandere ${ }^{2}$ \\ ${ }^{1}$ University of Latvia, Riga, Latvia \\ ${ }^{2}$ Rīga Stradiņš University, Riga, Latvia
}

\begin{abstract}
Low adherence to antihypertensive therapy is a major cause of adverse cardiovascular outcomes by uncontrolled high blood pressure. If the most frequent causes of inappropriate taking of medicine are identified, possible improvements within the health care could be performed, promoting the safety and efficacy of antihypertensive therapy. The aim was to find the factors affecting patient's adherence during antihypertensive therapy. In the period from June to September 2014 a quantitative study was carried out including patients with diagnosed arterial hypertension who visited pharmacies in Riga to get the antihypertensive therapy. The study collected data of 152 patients with the mean antihypertensive treatment duration of $9.7 \pm 2.4$ years. Most of the patients knew the reason for the use of the medication $(\mathrm{n}=123,80.8 \%)$, but it was also unclear for some patients $(\mathrm{n}=29,19.2 \%)$, more frequently with use of 2 or 3 medications at the same time, compared with the patients who received 4 or more medications $(\mathrm{n}=26,17.3 \%$ vs $\mathrm{n}=3,1.9 \% ; \mathrm{p}=0.063)$. High medication load was more frequently associated with lower adherence during antihypertensive therapy. Awareness of antihypertensive therapy for an individual patient, reminders of prescribed medications and dosage instructions may contribute to adherence of patients.
\end{abstract}

\section{Introduction}

Uncontrolled high blood pressure by incorrect and irregular use of medication is known to lead to severe complications even invalidity [1]. The World Health Organization (WHO) and studies reported that optimal antihypertensive treatment can reduce the risk of stroke by $30-40 \%$ and myocardial infarction by $15 \%$, meanwhile also prevent other chronic diseases [1-3].

One of important determinant of uncontrolled hypertension is low adherence to antihypertensive treatment. As a result, $50-75 \%$ of patients with arterial hypertension do not reach optimal blood pressure according to antihypertensive treatment guidelines $[4,5]$. Suboptimal blood pressure significantly increases the global health care costs and affects clinical outcomes [6]. All low adherence contributing factors can be divided into patient related factors and doctor related factors.

Patient related factors are age, gender, family and economic status. Most common doctor related factors include communication skills, knowledge of language and skill of implement royalty. Such health care system associated factors as distance to doctor's praxis and pharmacy, and system of medication compensation also can effect adherence $[4,7,8]$.

Low adherence to antihypertensive therapy is the major cause of adverse cardiovascular outcomes caused by uncontrolled high blood pressure. If the most frequent causes of 
Table 1. Understanding the use of medications according to its total count.

\begin{tabular}{|l|c|c|c|c|}
\hline $\begin{array}{l}\text { Number of } \\
\text { medications, } \mathbf{n}\end{array}$ & $\mathbf{1}$ & $\mathbf{2}$ or $\mathbf{3}$ & $\geq \mathbf{4}$ & \multirow{2}{*}{ p value } \\
\cline { 1 - 4 } Characteristics of knowledge of used medications \\
\cline { 1 - 4 } Clear, $\mathrm{n}(\%)$ & $44(28.8)$ & $64(42.3)$ & $15(9.6)$ & \multirow{2}{*}{$\mathbf{0 . 0 6 3}$} \\
\hline Unclear, $\mathrm{n}(\%)$ & 0 & $26(17.3)$ & $3(1.9)$ & \\
\hline
\end{tabular}

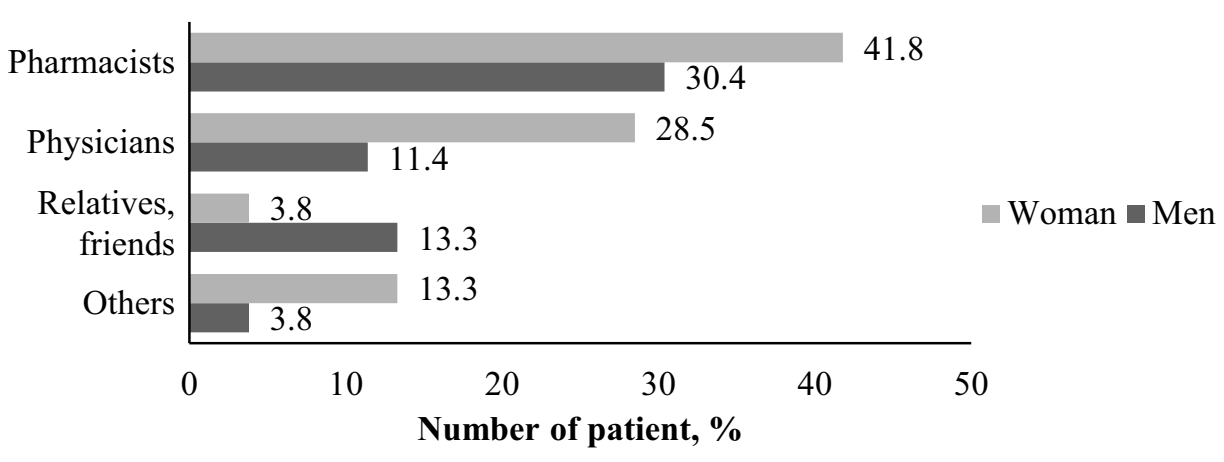

Figure 1. Sources of information on therapy of medications.

inappropriate use of medicine are identified, possible improvements within the health care plan could be performed, promoting the safety and efficacy of antihypertensive therapy from time of treatment beginning and also in the long term [3,9]. The aim of the study was to find the factors affecting patient adherence during antihypertensive therapy.

\section{Materials and methods}

In the period from June to September of 2014 quantitative study was carried out including patients with diagnosed arterial hypertension who visited pharmacies in Riga to get the antihypertensive therapy. Participation in the study was voluntary, filling the questionnaire. Basic characteristics of each patient were recorded - gender, age, education - as well as information on medications (names and total count), regime of use of the prescribed therapy, sources of information on medications and possible adverse reactions were collected.

\section{Results}

The study collected data of 152 patients (median age $61.4 \pm 10.7$ years) with the mean antihypertensive treatment duration of $9.7 \pm 2.4$ years. Most of the patients knew the reason for the use of the medication $(n=123,80.8 \%)$, but it was also unclear for some patients $(\mathrm{n}=29,19.2 \%)$, more frequently with the use of 2 or 3 medications at the same time, compared with patients who received 4 or more medications $(n=26,17.3 \% ; p=0.063$; Table 1).

Most often information on the use of medications patients, especially women, received from pharmacists $(n=110 ; 72.2 \%)$. Other sources of information on therapy are shown in Fig. 1. Men often took into account the views of friends and relatives about the use of medications.

The instructions of medications are always read only by $26(17.3 \%)$ patients, more rarely they read them regularly $(n=97,63.5 \%)$ or not at all $(n=29,19.2 \%)$, due to the complex 


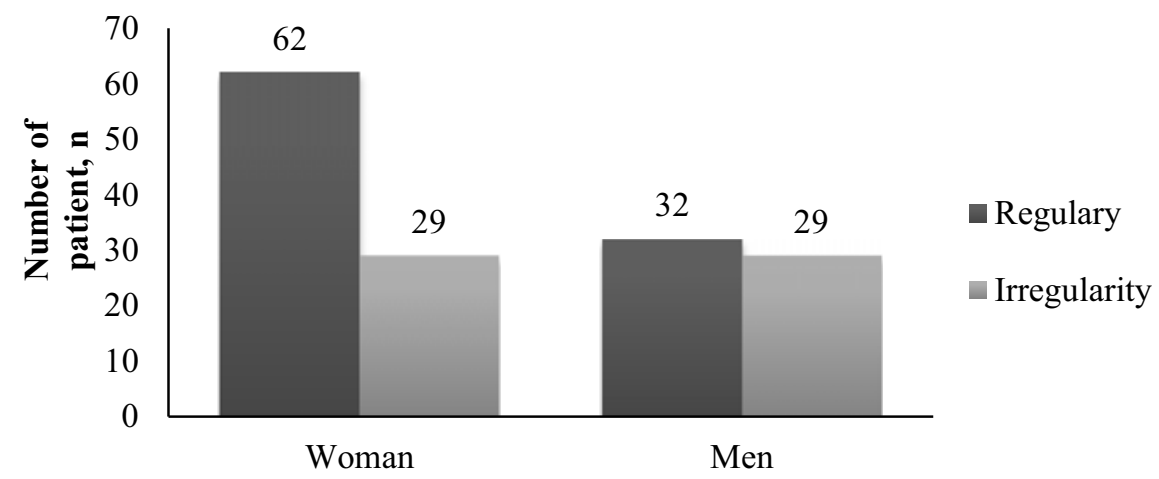

Figure 2. Characteristics of the use of medications depending on the gender of patients.

content of them $(n=50 ; 32.6 \%)$. About one-third of patients $(n=44,28.8 \%)$ answered that they were not informed about the adverse reactions of medications.

Most of the patients knew name and dosage of the prescribed medications accurately $(\mathrm{n}=97,63.5 \%)$. One-third of the studied patients named incorrectly their antihypertensive therapy $(n=44,28.8 \%)$, more frequently those who received 2 or 3 medications at the same time, compared with patients whose therapy consisted of less or more medications $(\mathrm{n}=3,1.9 \%, \mathrm{n}=15,9.6 \% ; \mathrm{p}=0.003)$. Difference between gender and habits of use of the prescribed therapy are displayed in Fig. 1. Women more often than men followed the medication treatment plan prescribed by the doctor.

More than one-fifth of the patients $(\mathrm{n}=35,23.1 \%)$ made changes in regime and doses of therapy themselves by choosing smaller $(n=21 ; 13.5 \%)$ or greater $(n=3 ; 1.9 \%)$ dose than recommended.

\section{Discussion}

Our data suggested that unclear use of medication promoted low adherence during antihypertensive treatment. In addition, other recent studies have showed that lack of knowledge of the development of the disease and serious consequences, as well as influence of medication, are significant factors, which considerably reduced patient's awareness of necessity to control the disease [4]. Especially in long term studies it was highlighted, which of the results confirmed lower adherence to long term treatment despite possible progression of the disease [4, 8]. Our research included also patients who used antihypertensive medications for long time, which explained our findings that both genders equally often made changes in regime and dose of therapy themselves, respectively used smaller or greater dose than recommended, according to their feelings. Such kind of behaviour promoted problems with control of optimal blood pressure, which could bring to enhancing of number of medications to facilitate therapy efficiency.

Our data showed that at this moment the greatest part of patients already used two or more anti-hypertensive medications therefore increase in number of medications also could increase the risk, that patients could change regime and dose of therapy by themselves and stop using some of the prescribed medications. In other study this information confirmed that lower adherence was associated with more prescribed medications [10], especially in users of diuretics and beta blockers, rarely in users of angiotensin - converting enzyme inhibitors (ACEI) and angiotensin II receptor blockers (ARB) [11], which was promoted by 
good tolerance of medication and availability in pharmacy [12]. An important fact from other studies was that one pill combination with two or more active medications substances more improved adherence during anti-hypertensive treatment $[9,13]$.

Alsabbagh et al. showed significant correlation of low adherence in therapy and patients' socio-economic conditions [7]. In our study this information was not described, although co-payment was high enough for medication purchase, which partly could provoke purchase of the prescribed medications. Regardless cheaper analogues of medications in pharmacy, patients did not always agree with change to them, being afraid of possible side effects or having doubts about medication efficiency offered by a pharmacist. Although our results reflected that most often patients received information on the use of medications from pharmacists, but in medication replacement they more trusted in doctor's suggestions. Situations when a patient got new medication with identical active substance and dosage, still could provoke extra rootless feelings and contribute to lowering of adherence [14]. Education of patients could be one of the most effective and costless available arrangements to prevent greater result of adherence during anti-hypertensive treatment. Other studies also show that lack of knowledge and information about medications were associated with low adherence $[15,16]$. As solutions to improve adherence were offered the following intelligible scheme of therapy, more information about the development of effect and possible side effects of the medication [17]. Similar to other studies also our data suggested that especially older men suspected lower adherence in treatment of prescribed medications [18]. As solution was suggested to choose medication with longer elimination of half period in this situation, which could allow patients with low adherence to reach optimal therapeutic efficiency [19].

Other study proved significant increase of adherence, when patients were motivated to control blood pressure every day at home [20]. Also pharmaceutical care system in Latvia provided possibility to control blood pressure in pharmacies which relieved monitoring of anti-hypertensive treatment efficiency. In this situation pharmacists had chance to take part in therapeutic improvement and could inform patients on necessity of medication, and explain unclear issues and give recommendations.

The main limitation of our study was small number of patients with a long term antihypertensive treatment.

\section{Conclusions}

High medication load was more frequently associated with lower adherence during antihypertensive therapy caused by insufficient knowledge of the received treatment. Pharmacists as health care professionals was an important source of information on medications therapy from patient's point of view. More often men than women made therapy corrections themselves during long term treatment. Awareness of antihypertensive therapy for individual patient, reminders of prescribed medicines and dosage instructions may contribute adherence of patients.

\section{References}

[1] M. Lemstra, M. W. Alsabbagh, Patient Pref. Adh. 8 (2014).

[2] World Health Organization. Adherence to long-term therapies (2003).

[3] K. Gordon, F. Smith, S. Dhillon, Patient Educ. Couns. 65 (2007).

[4] M.Chrostowska, K. Narkiewicz, Curr Vasc Pharmacol. 8 (2010).

[5] J. Jayasinghe, J. Cardiovasc. Nurs. 19 (2009). 
[6] T. A. Gaziano, A. Bitton, S. Anand, M. C. Weinstein, J. Hypertens. 27 (2009).

[7] M. H. Alsabbagh, M. Lemstra, D. Eurich, L.M. Lix, T.W. Wilson, E. Watson, D.F. Blackburn, Value Health 17 (2014).

[8] R. H. Chapman, A. A. Petrilla, J. S. Benner, J.S. Schwartz, S.S. Tang, Drugs Aging. 25 (2008).

[9] C. V. Ram, Manag. Care 22 (2013).

[10] C. I. Coleman, B. Limone, D. M. Sobieraj, S. Lee, M. S. Roberts, R. Kaur, T. Alam, J. Manag. Care Pharm. 18 (2012).

[11] I. M. Kronish, M. Woodward, Z. Sergie, G. Ogedegbe, L. Falzon, D. M. Mann, Circ. 123 (2011).

[12] C. D. Evans, D. T. Eurich, X. Lu, Y. M. Shevchuk, D. Blackburn, Am. J. Hypertens. 26 (2013).

[13] J. M. Neutel, Curr. Med. Res. Opin. 24 (2008).

[14] J. L. Lenahan, D. M. Mccarthy, T. C. Davis, L. M. Curtis, M. Serper, M. S. Wolf, J. Health Commun. 18 (2013).

[15] D. M. Cutler, W. Everett, N. Engl. J. Med. 362 (2010).

[16] W. J. Elliott, J. Clin. Hypertens. (Greenwich) 11 (2009).

[17] K. Traynor, Am. J. Health Syst. Pharm. 66 (2009).

[18] L. Osterberg, T. Blaschke, N. Engl. J. Med. 353 (2005).

[19] K. S. Ingersoll, J. Cohen, J. Behav. Med. 31 (2008).

[20] E. Marquez-Contreras, N. Martell-Claros, V. Gil-Guillen, J. J. Casado-Martínez, J. L. Martin-de Pablos, M. Figueras, J. Galera, A. Serra, J. Hypertens. 24 (2006). 